\title{
Paper
}

\section{Proposal of prediction accuracy improvement in non-invasive blood glucose measurement using MHC method}

\author{
RYO TAKEUCHI ${ }^{*}$ \\ Non-member, \\ KAZUHIKO NAGAO $\$$ Non-member
}

(Received July 30, 2020, revised December 29, 2020)

\begin{abstract}
According to the World Health Organization, 422 million adults worldwide have diabetes. Diabetic patients must regularly measure and manage their blood glucose levels. However, existing blood glucose meters require needles to draw blood, causing pain and infection problems. The measuring instrument to solve this problem would be a non-invasive blood glucose measuring instrument, but none is practically used at present. The authors have been studying non-invasive blood glucose measurement methods to reduce the burden on diabetic patients. As a result of previous research, we developed a simple non-invasive blood glucose meter and a blood glucose control system. Currently, our aim is to improve accuracy of blood glucose level prediction. In this paper we report on the blood glucose level prediction technique realized by using biological information. As a result, heart rate and MHC method are used as input data, and prediction using machine learning can be performed with high accuracy.
\end{abstract}

Keywords: blood glucose, non-invasive, $\mathrm{MHC}$,

\section{Introduction}

Diabetes is one of the top 10 causes of death in the world. The WHO reported that 422 million adults worldwide have diabetes [1]. The number of diabetics in Japan in 2014 was 3,166,000, an increase of 466,000 from three years before. In addition, as shown in Fig. 1, the number of people strongly suspected of having diabetes has been increasing since 1997 [2], and it is predicted that the number of patients with diabetes will continue to rise in the future. The treatment of patients with diabetes aims to prevent development and aggravation of complications characteristic to diabetes and accompanying diabetes, and to maintain the same quality of life as of healthy people [3]. In the management of diabetes mellitus, the blood glucose level must be monitored continuously.

Currently, self-monitoring blood glucose meters that can measure blood glucose levels at home are on the market. By using such a device, the blood glucose level can be repeatedly checked. However, current blood glucose selfmonitoring devices acquire a blood sample from the fingertip with a needle and measure it. This causes various problems [4]. A blood sample is obtained with a needle, which is painful and stressful to the patient. Also, needles and test strips are disposable, so they cost money and can cause infections during the blood collection. In addition, when continuous blood glucose measurement is performed in the management of diabetes mellitus, inflammation may

\footnotetext{
* Corresponding: takeuchi.ryo182@mail.kyutech.jp

$\dagger$ Kyushu Institute of Technology

$¥$ Department of Information Engineering, National College of Technology, Yuge Shosen High School.
}

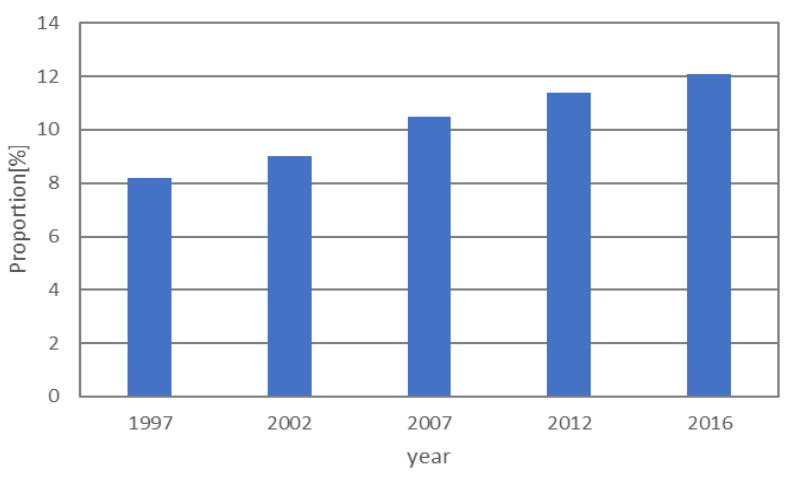

Figure 1: Trends in people suspected of being strongly susceptible to diabetes [2].

occur in the blood sampling area because blood glucose measurement is required several times a day depending on the severity. To solve these problems, minimally invasive blood glucose meters have been developed. For example, a FreeStyle Libre, developed by Abbott Diabetes Care Inc. USA is available. However, the false-positive rate of hypoglycemia remains high [5]. Although it is minimally invasive, the use of needles does not solve the problems of pain and infection. In order to completely solve these problems, there is a need for an inexpensive measuring instrument that does not require needle replacement or test paper exchange for each measurement, that can be easily used at home, and does not require blood sampling, thus not causing any sanitary problems. Such a measuring instrument is called a non-invasive blood glucose measuring instrument, however, none is practically used at present. 
Table 1: Comparison of noninvasive blood glucose measurement methods [12] [13].

\begin{tabular}{cccc}
\hline Method & Optical method & MHC technology & Smart lens(Google) \\
\hline Invasiveness & Noninvasive & Noninvasive & Noninvasive \\
Measurement object & Blood glucose & Metabolic heat & Glucose \\
Measurement accuracy & High & Low & Low \\
Medical waste & None & None & None \\
Production state & Production of prototype & Difficult as medical equipment & Failure \\
Size & $20 \times 10 \times 5 \mathrm{~cm}$ & Hand size & Contact lens \\
\hline
\end{tabular}

The importance of glycemic control not only in patients with diabetes but also in medical facilities is attracting attention. In Berghe et al. study [6], controlling blood glucose levels to $80 \sim 110 \mathrm{mg} / \mathrm{dL}$ in patients in intensive care units (ICU) reduced mortality from $8.0 \%$ to $4.6 \%$. It was also confirmed that the reduction of complications was achieved. In addition, the American Society of Intensive Care states that it is desirable to control blood glucose levels below $150 \mathrm{mg} / \mathrm{dL}$ as a treatment policy for sepsis and shock associated with sepsis [7]. Thus, awareness of the importance of glycemic control in ICUs has increased and has been discussed in related societies. In recent years, with the modernization of hospitals, IOT has been used for ehealth, telemedicine, human care, and patient monitoring health services [8] [11]. However, there is currently no adequate blood glucose monitoring system, and blood glucose levels are measured every few hours in clinical settings. It takes a lot of time and effort including prevention of infectious diseases during the blood collection. Providing a blood glucose meter and a control system that can operate non-invasively and continuously, would be a great medical advance.

The theory of noninvasive blood glucose measurement has been studied for many years. Table 1 compares noninvasive glucose monitoring methods [12] [13]. Previous studies have attempted to measure blood glucose using optical methods [14] or temperature methods [15]. In the case of optical methods, the size of apparatus is $20 \times 10 \times 5$ $\mathrm{cm}$ without including the power supply. On the other hand, if a method using temperature is used, it is possible to develop a small measuring instrument that can be mounted on a wearable terminal. This method, called Metabolic Heat Conformation (MHC), is based on the correlation between glucose concentration in the body and the amount of heat released by the body [15].

In this research, we aim to develop a non-invasive blood glucose meter and blood glucose management system that can be used at home. Conventional MHC theory uses blood flow to compensate for predictive values [15]. However, measuring blood flow velocity requires expensive, large instruments. That is, to measure blood glucose levels at home, prediction methods that do not require correction by blood flow velocity are required. As a result of previous research, we developed a simple non-invasive blood glucose meter and a blood glucose control system [16]. A prediction model was also studied to improve measurement accuracy [17]. However, previous studies have found that the predic-

IIAE Journal, Vol.9, No.1, 2021 tion accuracy of MHC method is poor. In this study, our goal is to achieve more accurate glucose level prediction by improving prediction methods and predicting blood glucose levels with consideration of biological information. In this paper, we report the results of a study on the prediction of blood glucose levels by considering multiple parameters of the human body in consideration of individual differences in biological responses. We also introduce new parameters that have not been used in MHC theory.

\section{Theory}

According to a previous study by Stephonie et al. [15], the appropriate sites for noninvasive glucose monitoring are the skin, forearm, wrist, earlobe, and fingertips. The parameters required for MHC method are heat radiation and heat convection. Heat radiation can be calculated by Stefan Boltzmann's law and is determined as Eq. 1.

$$
h r=\rho \times \sigma \times\left(T s^{4}-T o^{4}\right)
$$

$h r\left(W / m^{2}\right):$ radiation

$\rho$ : reflectivity of the skin's surface

$\sigma$ : Stefan Boltzmann constant

$T s\left({ }^{\circ} \mathrm{C}\right)$ : measured surface temperature

To $\left({ }^{\circ} \mathrm{C}\right)$ : measured ambient temperature

The thermal convection is obtained as Eq. 2 .

$$
h c=h \times(T s-T o)
$$

$h c\left(W / m^{2}\right):$ thermal convection

$h$ : convection coefficient

The equation for calculating the blood glucose level was derived by linear regression analysis based on the Eq. 1 and Eq. 2, and the blood glucose level was measured by an existing blood glucose meter. As an example, the following is the result of deriving the prediction equation. The data used in linear regression analysis are recorded blood glucose levels and fingertip temperature of an adult male at the ambient temperature of $28^{\circ} \mathrm{C}$. The sample size is 8 measurements. As a result, the calculation formula of blood glucose level was derived as Eq. 3 .

$$
\begin{aligned}
& \text { Glucose Level }(\mathrm{mg} / \mathrm{dL}) \\
& =193.17+h r+(-96.3)+h c \times 121.9
\end{aligned}
$$

MHC method is based on the correlation between glucose concentration in the body and the amount of heat released by the body. In a previous study by Stephonie et al. [15], the correlation between the glucose concentration and the amount of heat released by the body was 0.925 . 


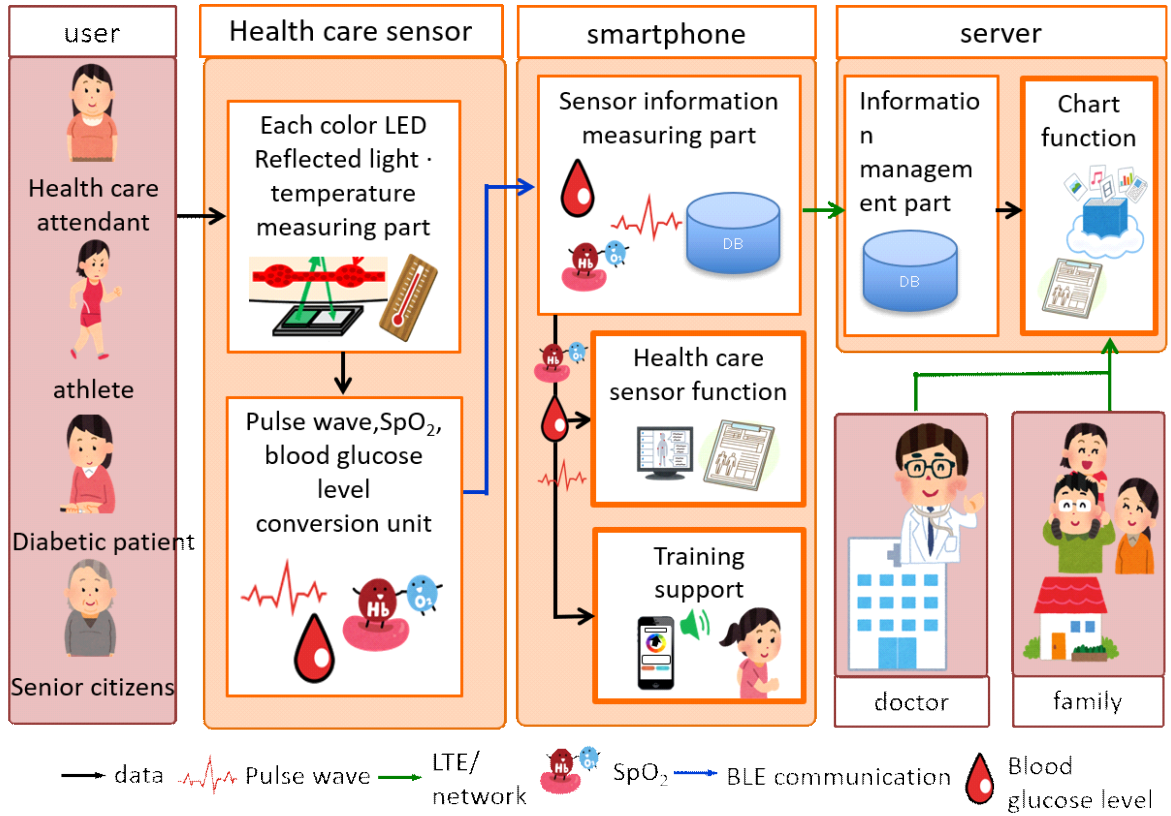

Figure 2: System configuration.

Table 2: Outline of the experiment data.

\begin{tabular}{ccccc}
\hline Experiment Number & Duration & Number of Subjects & Number of Samples & Remarks \\
\hline $\mathrm{A}$ & $12 / 2017 \sim 6 / 2018$ & 76 & 136 & - \\
$\mathrm{B}$ & $11 / 2018$ & 116 & 116 & - \\
$\mathrm{C}$ & $3 / 2019$ & 59 & 73 & - \\
$\mathrm{D}$ & $11 / 2020$ & 1 & 18 & before and after meals \\
\hline
\end{tabular}

\section{System Configuration}

The purpose of the proposed system is to measure blood glucose level and control blood glucose level easily even at home. Figure 2 shows the system configuration. It is mainly divided into a non-invasive blood glucose meter to measure the blood glucose levels and a smartphone application to manage the blood glucose levels. The details are explained in previous studies [16], and are therefore omitted here.

\section{Study of Blood Glucose Level Prediction Method}

In this study, the measurement experiments using a noninvasive blood glucose meter, accuracy evaluation, and blood glucose prediction models were investigated. Section 4.1 describes the evaluation criteria used in the accuracy evaluation. In Chapter 4.2, we report on the experiments and accuracy evaluation.

4.1 Experimental data The experimental data used in this paper are summarized in Table 2. The items in the Table 2 are as follows:

- Experiment Number

A number to be used in the following chapters to refer to the experimental data and contents

- Duration

The duration of the experiment

- Number of Subjects

The number of participants in the experiment
- Number of Samples

The number of samples obtained in the experiments

- Remarks

Special instructions are given.

Since the experiment may involve medical practice, safety considerations were implemented. The timing and frequency of data collection were determined by the subject at their own will. In addition, this research was conducted based on the approval of the FY 3010 Shikoku National College of Technology Bioethics Committee and the review of research on human subjects conducted by the Graduate School of Bioengineering, Kyushu Institute of Technology (FY 2019 5th Committee).

4.2 Error Grid Analysis The accuracy evaluation in this study was performed using the Error Grid Analysis (EGA) based on the International Organization for Standardization (ISO) standard. The EGA is used to evaluate the clinical accuracy of a blood glucose meter and can examine the clinical validity of a glucose monitoring system. The true value and the predicted value of the blood glucose level are shown as a scatter chart, and the reliability of the blood glucose measuring device can be evaluated in five zones. The definition of each zone is shown in Table 3. According to Table 3, the plotted data in zones A and B are of clinically acceptable accuracy. On the other hand, the values plotted beyond region $\mathrm{C}$ are inaccurate and clinically 
Table 3: Definition of EGA method.

\begin{tabular}{cl}
\hline Zone & Description \\
\hline A & The estimated blood glucose level deviates about $\pm 20 \%$ from the actual value \\
B & Self-monitoring device giving predictive blood glucose level degree results differ by more than $20 \%$ from actual value \\
C & Poor results of self-monitoring equipment \\
D & If detection fails, it is outside the range of actual value (70 to $180 \mathrm{mg} / \mathrm{dL})$ \\
E & False blood glucose level causing serious problems in diabetes management \\
\hline
\end{tabular}

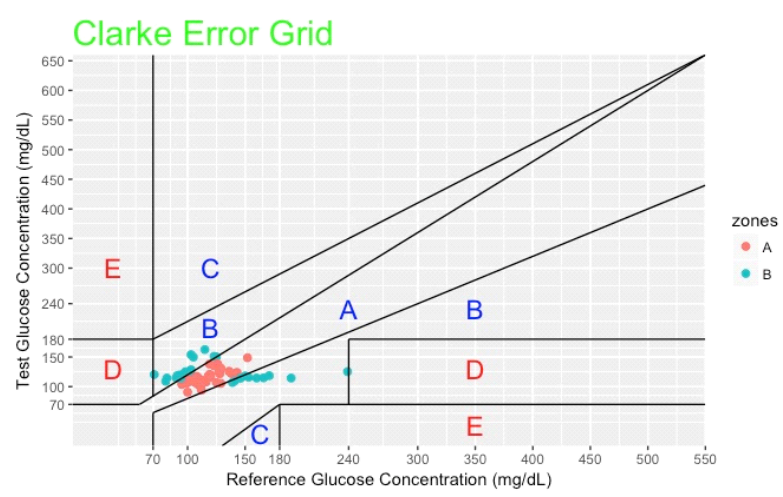

Figure 3: Linear regression analysis results.

unacceptable.

\subsection{Linear Regression Analysis VS Neural Network}

We have compared models for predicting blood glucose levels as a result of previous studies [17]. A linear regression analysis and a neural network were used for comparison. The neural network consists of three layers: an input layer, an intermediate layer, and an output layer. At this time, two parameters calculated from Eq. 1 and Eq. 2 and blood glucose levels measured by an existing blood glucose level measuring device were used as inputs for prediction. Half of the 136 data sets were used for learning and the other half were used for testing. The samples were taken between December 2017 and June 2018 (Table 2, Experiment Number A).

An example in Fig. 3 shows the result of prediction using a linear regression analysis, and Fig. 4 shows the result of prediction using a neural network. In the case of linear regression analysis, $61.2 \%$ of the values and $33.8 \%$ of the values are plotted in zone A and zone B, respectively. In contrast, when the neural network was used, $70.5 \%$ and $29.5 \%$ of the values were plotted in zones A and B, respectively. Thus, it was confirmed that the prediction accuracy can be improved by using neural networks.

\subsection{Influence of Bioinformatics on Glucose Prediction}

The predictions in previous studies [17] did not take into account differences in biological information. In this chapter we investigate the relationship between blood glucose levels and gender, age, weight, and height.

Firstly, the experiments were conducted to investigate the relationship between the abovementioned parameters and the blood glucose levels. In this experiment, the measurement experiments were carried out at the health examination of Ehime Bank, Inc. in November 2018 (Table 2, Ex-

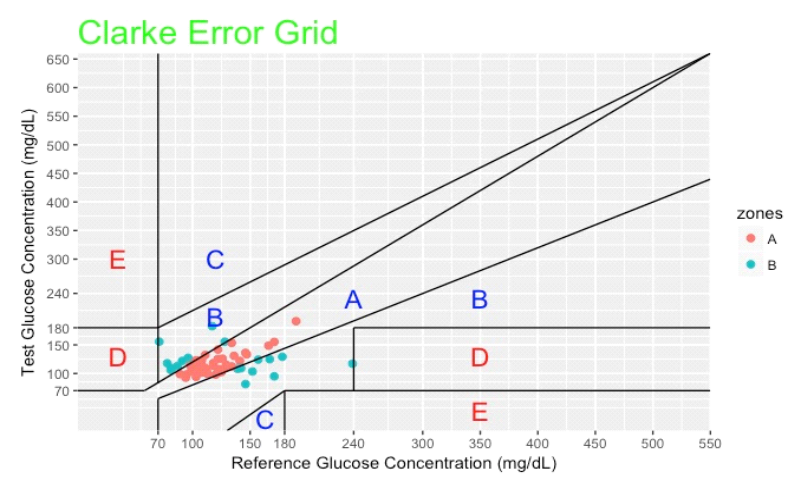

Figure 4: Prediction results by neural network.

Table 4: Correlation coefficient between blood glucose level and each parameter.

\begin{tabular}{cc}
\hline Parameters & Correlation Coefficient \\
\hline Age & 0.412 \\
Body weight & 0.214 \\
Hight & -0.0161 \\
\hline
\end{tabular}

periment Number B). The average blood glucose level was $96.38 \mathrm{mg} / \mathrm{dL}$ for females and $101.4 \mathrm{mg} / \mathrm{dL}$ for males, confirming that males had slightly higher average blood glucose levels. Table 4 shows the correlation coefficients between the blood glucose level and age, weight, and height. From these results, it was confirmed that there is a correlation between the age and weight and the blood glucose level, and that there is no correlation between the height and the blood glucose level. These results suggest that accuracy can be improved by predicting blood glucose levels with consideration of age and body weight.

Based on these results, the blood glucose levels were predicted in consideration of gender, age, and weight. The data used are 73 samples taken at the 32 Yumeshima Kaido Ikina Marathon held on March 3, 2019 (Table 2, Experiment Number C). Two parameters (thermal radiation and convection) and five parameters (thermal radiation, thermal convection, gender, age, and weight) were used for comparison to predict blood glucose levels. The results are shown in Fig. 5 and Fig. 6. In Fig. 5, it can be said that the prediction result is biased and the blood glucose level cannot be accurately predicted. However, in Fig. 6, 64.8\% of the values are plotted in zone A, $27.0 \%$ in zone B, $2.70 \%$ in zone C, and $5.4 \%$ in zone D. Although a few values are plotted in zones $\mathrm{C}$ and $\mathrm{E}$, most values were plotted in zones $\mathrm{A}$ and $\mathrm{B}$, and it was confirmed that the accuracy of blood glucose level 


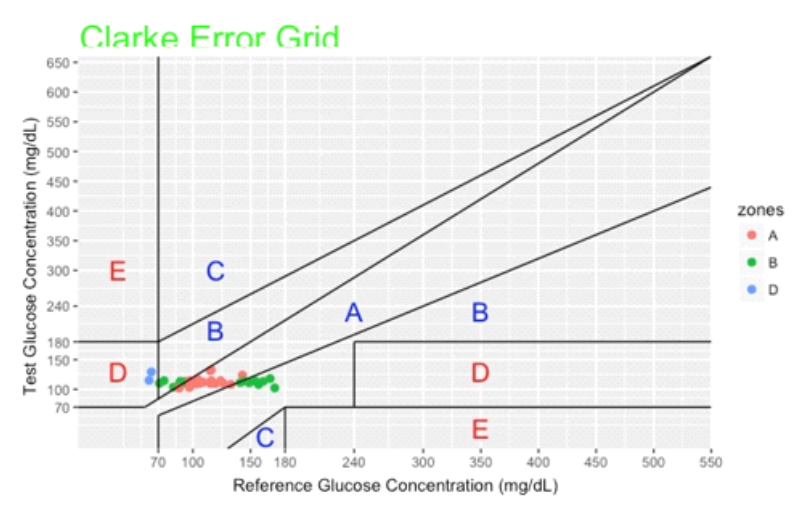

Figure 5: Prediction results (Parameter Type 2).

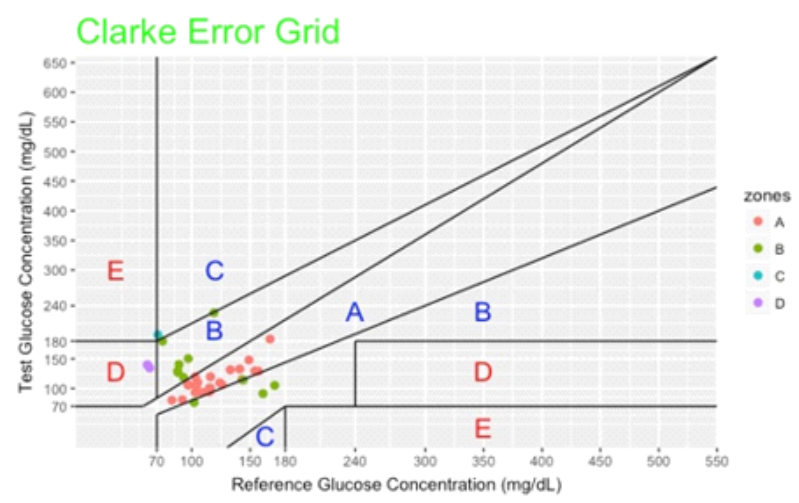

Figure 6: Prediction results (Parameter Type 5).

prediction was improved by considering the added parameters. The RMSE was $35.57 \mathrm{mg} / \mathrm{dL}$ when prediction was made using five parameters.

\subsection{Prediction of blood glucose levels, including heart} rate In this chapter, in addition to MHC techniques, we consider glucose prediction by introducing heart rate. From the results of previous studies [15] and up to the previous chapter, it has been confirmed that $h r$ and $h c$ are important values for prediction of blood glucose level. However, there are other factors that change the temperature of the fingertips besides the absorption of glucose in the body. Therefore, the current situation is considered to be the limit for accuracy to predict blood glucose levels. In addition to the MHC technique, we examined possibilities to add new parameters. A study by Kurasawa et al. [18] examined the prediction of fasting glucose by heart rate. Takai et al. [19] reported a significant difference in heart rate between healthy subjects and those with hyperglycemia. Therefore, it is considered that there is a correlation between the heart rate and the blood glucose level.

In this experiment, four parameters were obtained from the preprandial to postprandial blood glucose level, thermal radiation, thermal convection, and heart rate. We would like to monitor the variation progress of the blood glucose level until it goes down after a meal, but the number of times that the blood glucose level can be measured is limited because the existing blood glucose measuring instruments use needles. Studies by Esposito et al. [20] have generally reported that the peak postprandial hyperglycemia occurs 1
Table 5: Comparison with prediction including heart rate.

\begin{tabular}{ccc}
\hline & $h r, h c$, & $h r, h c, H R$ \\
\hline RMSE of glucose level(mg/dL) & 53.58 & 20.94 \\
\hline
\end{tabular}

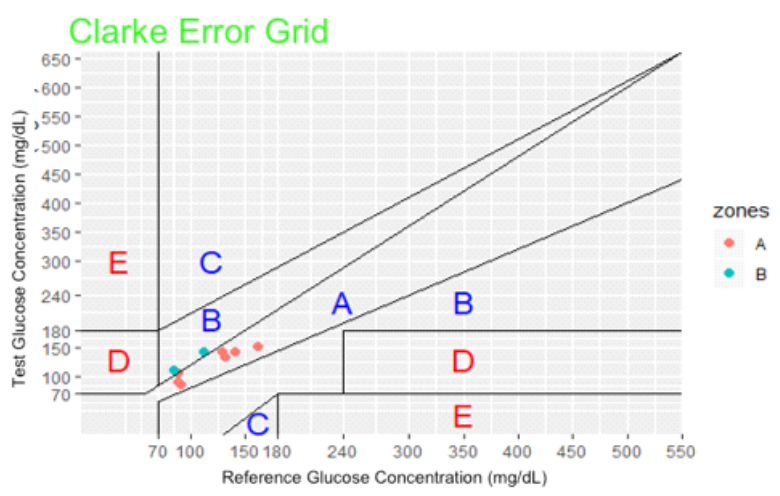

Figure 7: Forecast results (Prediction including heart rate).

hour after a meal when glycemic control is normal. Therefore, in this experiment, four measurements were performed for several days before meal, 30 minutes after meal, $60 \mathrm{~min}$ utes after meal, and 3 hours after meal. The sample data is 18 points from 1 subject (Table 2, Experiment Number D).

Based on these data, two types of predictions were made and compared: prediction using only heat radiation and heat convection, and prediction of blood glucose levels using heat radiation, heat convection, and heart rate. For comparison, cross-validation was performed and the model was evaluated using RMSE. The comparison results are shown in Table 5. From Table 5, it is confirmed that the accuracy of blood glucose level prediction can be greatly improved by adding heart rate. The results of accuracy evaluation using EGA are shown in Fig. 7. In Fig. 7, 77.8\% of the values were plotted in zone A and $22.2 \%$ in zone B. As the number of samples is small, it cannot be asserted, but based on the results of Table 5 and Fig. 7, it is confirmed that further improvement of prediction accuracy can be expected by adding heart rate to the blood glucose level prediction using MHC method.

\section{Conclusion}

This paper presents an improved prediction method based on MHC method. In particular, the prediction using neural network and the possibility of adopting new input parameters were verified.

We compared linear regression analysis and neural network as glycemic prediction models. By applying the neural network, the accuracy improvement of about $10 \%$ was realized in comparison with the method using the conventional linear regression analysis. In addition, it was confirmed that the prediction accuracy drastically improved, when the glucose level prediction considering body information and biological information which were not considered in previous research was carried out. Especially, the glucose level was estimated from the relationship between heart rate and glucose level, and it was confirmed that $78 \%$ 
of the values were in zone A of the EGA. We found that the heart rate is an important parameter in predicting blood glucose level to compensate for the inaccuracies of conventional MHC method. In the future, the number of data will be increased, and we expect that the accuracy of the experiment will be further improved. The presented results may solve the problem of poor prediction accuracy in non-invasive blood glucose measurements based on MHC method. In addition, in the MHC theory, it has been shown that a blood glucose level prediction method that does not require measurement of blood flow velocity can be realized.

\section{Acknowledgment}

The authors would like to express gratitude to the participants of the 31 and 32 Ikina marathons, Ehime Bank, Ltd., Himegin Soft, Inc., and Angel, a social welfare corporation, for their cooperation in the measurement experiments in this study.

\section{References}

[1] World Health Organization, "GLOBAL REPORT ON DIABETES", 2016.

[2] Ministry of Health, Labour and Welfare, "Heisei 28 nen kokuminkenkou eiyoutyousakeltuka no gaiyou (in Japanese)", 2017.

[3] M. Haneda, M. Noda, H. Origasa, H. Noto, D. Yabe, Y. Fujita, A. Goto, T. Kondo and E. Araki, "Japanese Clinical Practice Guideline for Diabetes 2016", Diabetology International, Vol.9, pp.1-45, 2018. DOI: 10.1007/s13340-0180345-3

[4] Taku Takeda, "Keltutou ziko sokutei no gaiyou", The Journal of the Japanese Society of Internal Medicine, Vol.98, No.4, pp.761-767, 2009.

[5] A. J. Talib, M. Alkahtani, L. Jiang, F. Alghannam, R. Brick, C. L. Gomes, M. O. Scully, A. V. Sokolov and P. R. Hemmer, "Lanthanide ions doped in vanadium oxide for sensitive optical glucose detection", Optical Materials Express, Vol.8, No.11, pp.3277-3287, 2018. DOI: 10.1364/OME.8.003277

[6] G. V. D. Berghe, P. Wouters, et al., "Intensive insulin therapy in critically ill patients", The New England Journal of Medicine, Vol.345, No.19, pp.1359-1367, 2001. DOI: 10.1056/NEJMoa011300

[7] R. P. Dellinger, J. M. Carlet, H. Masur, et al., "Surviving Sepsis Campaign guidelines for management of severe sepsis and septic shock", Intensive Care Medicine, Vol.30, No.3, pp.536-555, 2004. DOI: 10.1007/s00134-004-2210-z

[8] A. H. Del'Aulnoit, S. Boudet, M. Génin, P. -F. Gautier, J. Schiro, D. H. del'Aulnoit and R. Beuscart, "Development of a Smart Mobile Data Module for Fetal Monitoring in E-Healthcare", Journal of Medical Systems, Vol.42, No.5, pp.83, 2018. DOI: 10.1007/s10916-018-0938-1

[9] A. A. Abdellatif, A. Emam, C. -F. Chiasserini, A. Mohamed, A. Jaoua and R. Ward, "Edge-based compression and classification for smart healthcare systems: Concept, implementation and evaluation", Expert Systems with Applications, Vol.117, No.1, pp.1-14, 2019. DOI: 10.1016/j.eswa.2018.09.019

[10] E. B. Ferlie and S. M. Shortell, "Improving the quality of health care in the United Kingdom and the United States: A framework for change", Milbank Quarterly, Vol.79, No.2, pp.281-315, 2003. DOI: 10.1111/1468-0009.00206

[11] H. Shen, D. Ma, Y. Zhao, H. Sun, S. Sun, R. Ye, L. Huang, B. Lang and Y. Sun, "MIAPS: A web-based system for remotely accessing and presenting medical images", Computer Methods and Programs in Biomedicine, Vol.113, No.1, pp.266-283, 2014. DOI: 10.1016/j.cmpb.2013.09.008

[12] Saiketu ga huyou, hisinsyuu keltutouti sensa no zituyouka ni tyousen (in Japanese), National Institutes for Quantum and Radiological Science and Technology, https://www.qst. go.jp/site/kansai-aqocg/, access date: 2020.12.22

[13] Z. Blum, D. Pankratov and S. Shleev, "Powering electronic contact lenses: Current achievements, challenges, and perspectives", Expert Review of Ophthalmology, Vol.9, No.4, pp.269-273, 2014. DOI: 10.1586/17469899.2014.922873

[14] M. C. Pande and A. K. Joshi, "Non-Invasive Blood Glucose Measurement", International Journal of Computational Engineering Research, Vol.5, No.4, pp.129-131, 2015.

[15] S. Y. H. Kit, "Non-Invasive Blood Glucose Measurement Using Temperature-based Approach", Jurnal Teknologi, Vol.64, No.3, 2013.

[16] R. Takeuchi, A. Seo and K. Nagao, "hisinsyuu keltutou sokuteiki no kaihatu oyobi seido tyousa (in Japanese)", Information Processing Society of Japan, Forum on Information Technology 2018, No.CO-004, pp.47, Fukuoka Institute of Technology, 2018.

[17] R. Takeuchi and K. Nagao, "MHC gizyutu wo motiita hisinsyuu keltutousokuteiki no kaihatu (in Japanese)", Information Processing Society of Japan, The 81 st National Convention of IPSJ, No.6ZG-08, pp.665-666, Fukuoka University nanakuma campus, 2019.

[18] K. Esposito, M. Ciotola, D. Carleo, B. Schisano, L. Sardelli, D. D. Tommaso, L. Misso, F. Saccomanno, A. Ceriello and D. Giugliano, "Post-meal glucose peaks at home associate with carotid initima-media thickness in type 2 diabetes", The Journal of Clinical Endocrinology \& Metabolism, Vol.93, No.4, pp.1345-1350, 2008. DOI: 10.1210/jc.2007-2000

[19] T. Takai, A. Matsuda, K. Saito, K. Yamamoto, Y. Sakamoto, T. Kuzuya, S. Yoshida and M. Ota, "Variations in Heart Rate During Deep Breathing as an Early Index of Diabetic Autonomic Neuropathy", Journal of the Japan Diabetes Society, Vol.26, No.1, pp.37-43, 1983.

[20] S. Kurasawa, S. Koyama, H. Ishizawa, K. Fujimoto and S. Chino, "Verification of Non-Invasive Blood Glucose Measurement Method Based on Pulse Wave Signal Detected by FBG Sensor System", Sensors, Vol.17, No.12, 2017. DOI: $10.3390 / \mathrm{s} 17122702$ 


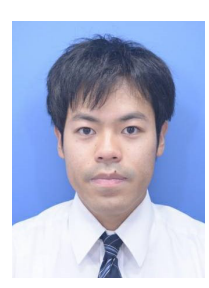

Ryo Takeuchi (Non-member) was born in 1996. He graduated from the Department of Electronic Control Engineering, Kitakyushu Technical College in 2017. In 2019, he completed the YUGE College of Commerce. In the same year, he entered the Master's course of the Graduate School of Kyushu Institute of Technology.

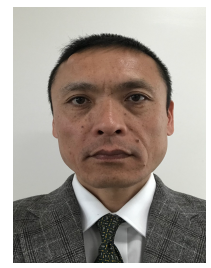

Kazuhiko Nagao (Non-member) was born in Kochi Prefecture in 1946. Completed the Master's course in Electrical and Information Engineering, Graduate School of Engineering, Nagoya Institute of Technology in 1989. In the same year, Assistant Professor, Department of Information Engineering, National College of Technology, Yuge Shosen High School. He became a professor in 2008. Engaged in practical engineering education through a technical college programming contest. Information Processing Society of Japan Award for Excellence in Education (2009), Education System Contest Award for Excellence (2010). Information Processing Society Board of Education Technical College Board of Education. He is a member of the Society of Electronics, Information and Communication Engineers, the Society of Artificial Intelligence, the Society of Educational System Information, and the Japan Society of Open Communication. Doctor of Engineering.

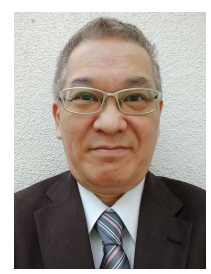

Hiroyuki Miyamoto (Non-member) was born in 1961. He graduated from Department of Systems Science, Graduate School of Engineering Science, Osaka University in 1987. He has been an Associate professor at Graduate School of Kyushu Institute of Technology since 2000. 